\title{
Challenges and Limitations of Narrow-Band Optical Imaging for Intra-Operative Parathyroid Identification
}

\author{
Laura M. Higgins ${ }^{\mathrm{a}}$, Mark C. Pierce ${ }^{\mathrm{a}, \mathrm{c}, \mathrm{d}}$, Tomer Davidov ${ }^{\mathrm{b}, \mathrm{c}}$
}

\begin{abstract}
Background: Surgical resection of the parathyroid glands can be challenging due to poor visual contrast between the parathyroid and surrounding tissues. We hypothesized that narrow-band imaging (NBI) could enhance subtle differences in the colors of these tissues for improved visual contrast during parathyroid surgery.

Methods: The diffuse optical reflectances of parathyroid, fat, muscle, and thyroid tissue were measured in vivo as a function of wavelength during six surgical procedures, using a fiber-optic probe and spectrophotometer. Analysis of these data led to three candidate NBI wavelength bands which exhibited the largest differences in reflectance between parathyroid and other tissue types. Color filters at these wavelength bands were attached to the surgeon's loupes for qualitative visual assessment, and also to a digital camera for quantitative assessment of tissue contrast in seven additional surgeries.
\end{abstract}

Results: Spectroscopic measurements showed that diffuse optical reflectance at green $(515-565 \mathrm{~nm})$, yellow $(570-613 \mathrm{~nm})$, and red (604 - $644 \mathrm{~nm}$ ) wavelengths showed the greatest difference in intensity (appearing brighter or darker) between parathyroid and surrounding tissues. However, visual and digital NBI of the parathyroid using these wavebands generated measured intensities which were not significantly improved as compared to white light, providing only minimal contrast enhancement for the surgeon.

Conclusions: Narrow-band color filters can be added to conventional loupes to selectively view the surgical field with light at specific wavelengths. When the parathyroid is viewed with filters chosen to maximize visual contrast between tissue types, there appears to be little benefit in contrast enhancement between tissues due to diminished

Manuscript accepted for publication March 08, 2016

aDepartment of Biomedical Engineering, Rutgers, The State University of New Jersey, Piscataway, NJ, USA

bDepartment of Surgery, Robert Wood Johnson Medical School, New Brunswick, NJ, USA

${ }^{\mathrm{c}}$ These two authors are co-senior authors.

${ }^{\mathrm{d}}$ Corresponding Author: Mark C. Pierce, Department of Biomedical Engineering, Rutgers, The State University of New Jersey, 599 Taylor Road, Piscataway, NJ 08854, USA. Email: mark.pierce@rutgers.edu

doi: http://dx.doi.org/10.14740/jcs295w overall brightness.

Keywords: Optical imaging; Surgical guidance; Intra-operative imaging

\section{Introduction}

Primary hyperparathyroidism is a disease in which one or more of the parathyroid glands become enlarged, secreting excess amounts of parathyroid hormone. This results in an elevated serum calcium level which may result in accelerated bone loss, bone pain, kidney stones, profound fatigue, and cognitive dysfunction, among other medical ailments [1]. Parathyroidectomy involves surgical removal of one or more parathyroid glands to restore serum parathyroid hormone and calcium levels to normal [2]. The location of an enlarged gland can be determined pre-operatively by a variety of well-known localization studies [3]. The most effective and widely used method is the ${ }^{99 \mathrm{~m}} \mathrm{Tc}$-sestamibi parathyroid nuclear scan $[3,4]$. Other localization studies include neck ultrasound [5], 4D CT [6] and selective venous sampling [7]. The surgeon's experience also plays an important role in parathyroid surgery and has been positively correlated with successful identification of parathyroid glands [8]. Similarly, clear identification of normal parathyroid tissue for preservation during thyroid surgery is essential to minimizing patient morbidity and other complications [9]. However, in both cases, visual intra-operative identification of the parathyroid gland can still be challenging for even the experienced endocrine surgeon due to the anatomic variability of the gland's location and its resemblance to neighboring tissues in the neck [10].

The goal of this study was to establish whether variations in natural tissue optical properties could be exploited to improve visual identification of the normal parathyroid gland during surgery. Previous work in this area has used techniques including elastic scattering spectroscopy [11-13], optical coherence tomography [14], and near-infrared (NIR) fluorescence imaging [15-18]. However, each of these methods requires a secondary display for feedback, requiring the surgeon to periodically look away from the surgical field and to interpret these images or spectra. Narrow-band imaging (NBI) uses a discrete range of optical wavelengths (colors) instead of broadband 

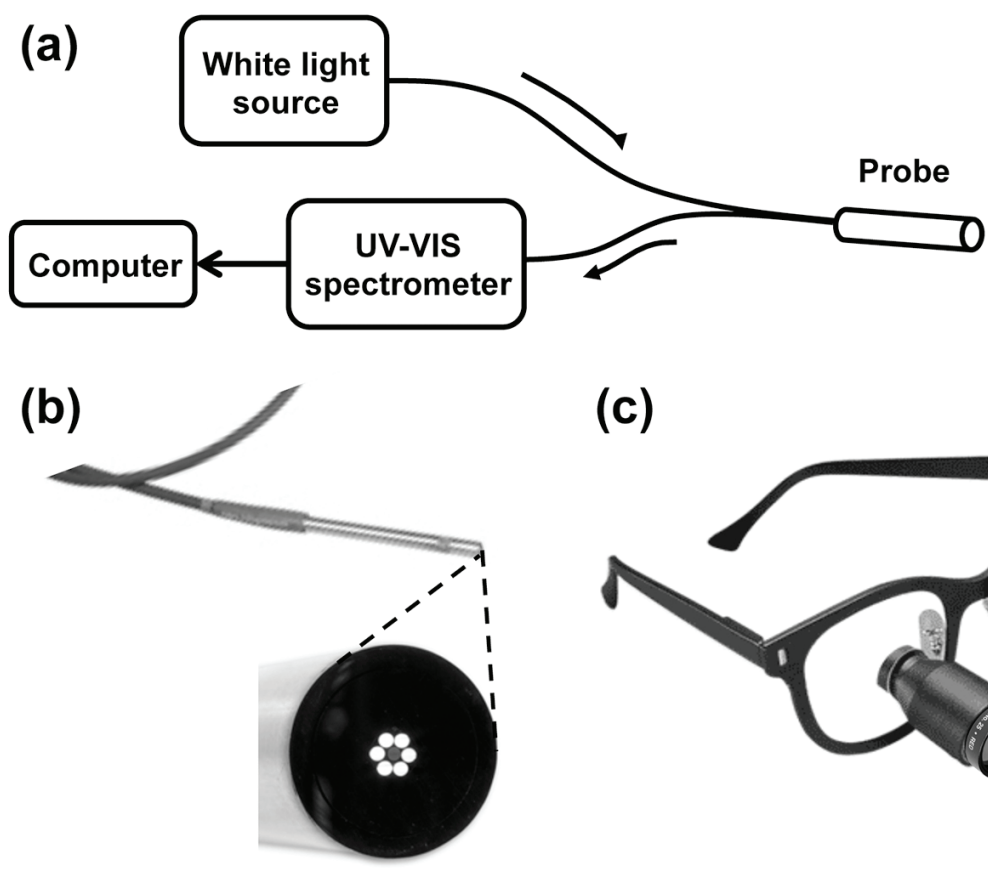

(c)

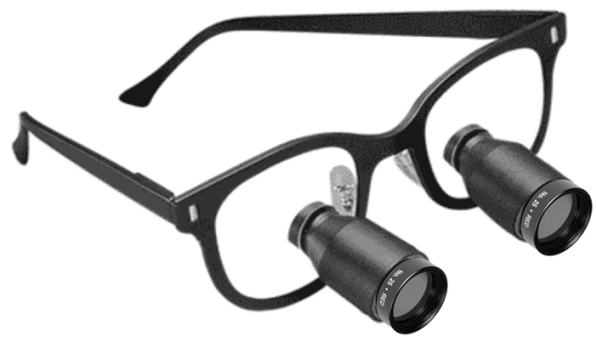

Figure 1. (a) Schematic diagram of the reflectance spectroscopy apparatus. (b) Photograph of the probe tip (3.175 mm (1/8") diameter) with magnified view of the probe tip end face, showing six illumination fibers surrounding a single light collection fiber. (c) Photograph of optical filters fixed to surgical loupes.

white light for viewing the tissue. NBI has previously been used to enhance intra-operative visualization of highly vascular tissue structures $[19,20]$ by using green color filters, corresponding to wavelengths where hemoglobin strongly absorbs light. NBI can be easily implemented in the surgical setting by using digital cameras with external filters, or more simply through direct visualization by eye. We hypothesized that NBI may increase the visual contrast between the parathyroid gland and surrounding tissues, leading to improved identification of the gland during surgical procedures.

To test this hypothesis, we performed a two-stage clinical study. First, we aimed to establish the optimum NBI wavebands (colors) for distinguishing parathyroid tissue from neighboring tissues by collecting diffuse reflectance spectroscopy measurements from these tissues in vivo. Second, using three candidate wavebands identified by the spectroscopic measurements, we implemented a simple color filter modification to standard surgical loupes and a digital camera to perform qualitative and quantitative NBI in an independent set of subjects.

\section{Materials and Methods}

Under approval from the Rutgers University Institutional Review Board, measurements for the study were taken during surgical procedures at Robert Wood Johnson University Hospital. Patients over 18 years of age undergoing partial or total thyroidectomy or parathyroidectomy were invited to participate in the study. After obtaining written informed consent, a total of 13 patients were enrolled in the study (three parathyroidectomy and 10 thyroidectomy).
In the first stage of the study, a fiber-optic probe (Ocean Optics, Dunedin, FL) was used to collect diffuse reflectance spectra from thyroid, parathyroid, muscle, and fat in each patient (Fig. 1a). The probe illuminates tissue with broadband white light from a tungsten-halogen source transmitted through six concentric optical fibers. Light reflected or backscattered from the tissue is collected by one central optical fiber (Fig. 1b) and delivered to a spectrophotometer (Ocean Optics). With this light source and spectrometer, we were able to measure the reflectance over a range of $360-900 \mathrm{~nm}$. Prior to the intraoperative measurement procedure, a spacing collar was placed around the reflectance probe tip to ensure that the distance between the probe end face and the tissue was constant for all measurements (about $40 \mathrm{~mm}$ ). The fiber-optic probe was placed in a sterile ultrasound sheath for measurements. Following visual identification of each tissue type based on the surgeon's visual impression, the probe's white light source was turned on and the surgical overhead lights were turned off to avoid contributing to the measured reflectance signal. The probe was then placed directly onto the tissue surface to measure the light reflected under white light illumination. Five separate reflectance spectra were acquired from each tissue type in each subject. Measurements were also acquired from a calibrated diffuse reflectance target (Spectralon) and a dark reference, to account for any source non-uniformity or effects of the ultrasound sheath. Post-operatively, each set of five sample spectra were combined to obtain a mean reflectance spectrum for each tissue type in each subject. These averaged spectra were then smoothed using a Savitzky-Golay filter and normalized using the method described by Johnson et al [21], whereby the mean intensity of each spectrum was subtracted 

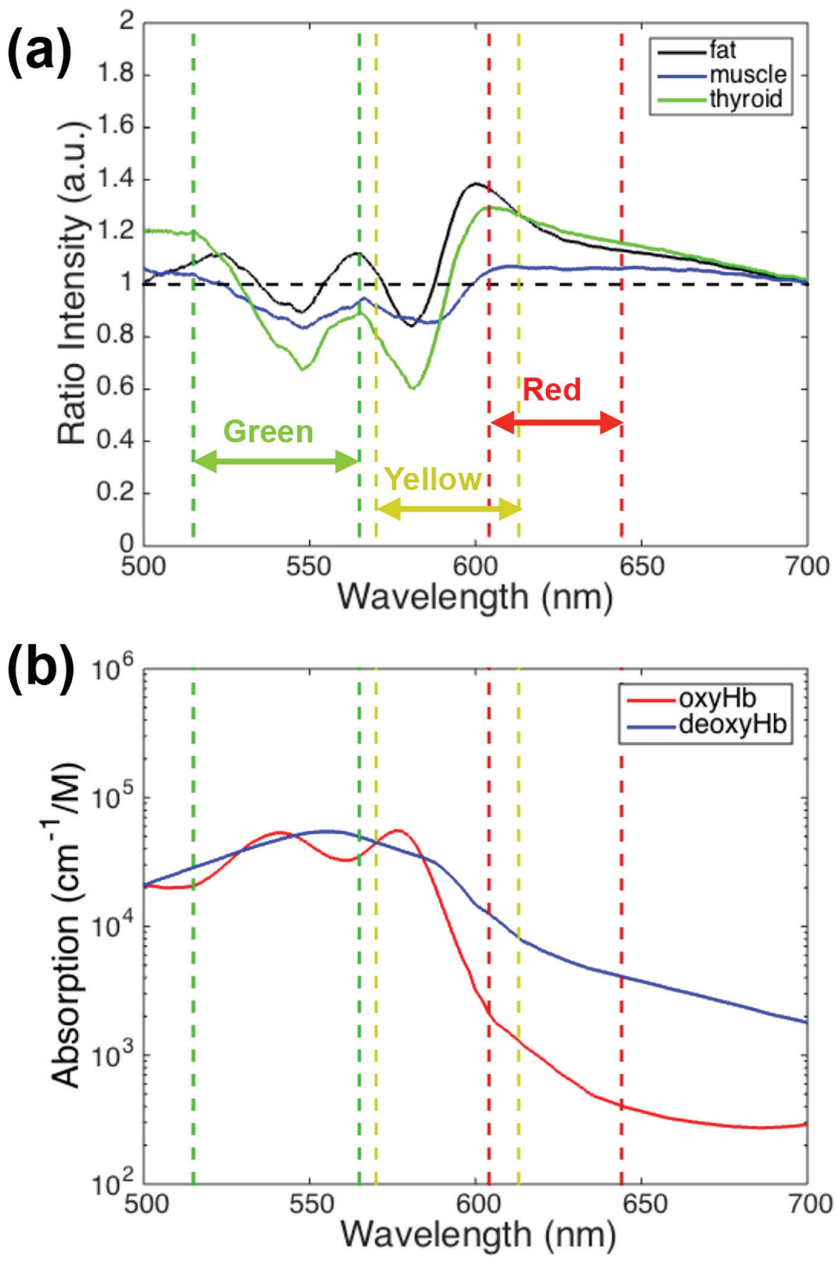

Figure 2. (a) Mean reflectance ratio of thyroid, fat, and muscle relative to parathyroid tissue. The dashed lines and arrows correspond to the narrow band filters selected for the imaging stage of the study. (b) Oxyand deoxy-hemoglobin absorption as a function of wavelength.

from each data point. This process results in each spectrum representing the relative reflectance of tissue at each wavelength, independent of the absolute light intensity level. The ratio of the normalized reflectance spectrum intensity for the parathyroid gland to each of the other tissues was calculated to determine the wavelengths (colors) at which the largest reflectance differences occur. Using these spectroscopy results, three commercially available optical band-pass filters were selected in the identified regions in order to enhance the differences (contrast) between the tissues for the second stage of the study.

The ability of each color filter to improve visual identification of the parathyroid gland was then evaluated. After the surgeon identified the parathyroid gland under standard operating room lights, the appearance of each tissue type was evaluated when viewed through each color filter. Using a custom designed mount, two 1-inch diameter filters were attached to the surgeon's loupes (Fig. 1c) to enable visual examination using only light reflected in each of the selected wavelength bands. Each wavelength band was scored on a scale from 1 to 5 , based on the surgeon's qualitative assessment in distinguishing the parathyroid gland from the surrounding tissue. Narrow-band digital imaging was also performed for quantitative evaluation, by mounting a separate 2-inch diameter filter onto the lens of a digital camera (Nikon D3200), for each of the three wavebands tested. Two digital images of the field were taken using each filter. Finally, an additional white light image was taken of the surgical field for comparison with narrow-band filtered images.

After surgery, the surgeon manually outlined each tissue type on the white light image of the field. The corresponding locations were mapped to each of the NBI images. The mean grayscale pixel values for the parathyroid, thyroid, muscle, and fat regions were calculated from each filter image and these results were used to evaluate the effectiveness of each filter in distinguishing the parathyroid glands from the surrounding tissue.

\section{Results}

A total of 13 subjects participated in this study. All were female with a mean age of 52 years (range: $31-83$ ). Spectroscopy measurements were performed on all 13 subjects and image data were collected from subjects 7 to 13 only. The initial subjects in each group were used to optimize the respective measurement procedure (spectroscopy or imaging) and data from these patients were not included in analysis. Overall, spectroscopy data from patients 5 to $13(n=9)$ and image data from patients 10 to $13(n=4)$ were included in the subsequent analysis. Of the procedures included in data analysis, one was a parathyroidectomy and eight were thyroidectomies. Healthy tissues were measured when possible. All parathyroid spectra and images were taken of normal parathyroids $(n=9)$. For the patients undergoing partial thyroidectomy $(n=2)$, the remaining, healthy lobe was measured. Measurements were performed on patients undergoing total thyroidectomies $(n=5)$ prior to the removal of the gland.

Figure 2a shows the mean reflectance of each tissue type relative to the parathyroid gland, over a $500-700 \mathrm{~nm}$ wavelength range, where the largest differences were visible. The horizontal dashed black line corresponds to a ratio of one, indicating where the reflectances (brightness) of the parathyroid gland and other tissue type are equal. Tissue that appears brighter than the parathyroid gland at a particular wavelength has a ratio greater than 1 while tissue that appears darker than the parathyroid gland has a ratio less than 1 . The ratios for each tissue type have local minima around 548 and $580 \mathrm{~nm}$ with a local maximum near $564 \mathrm{~nm}$. Optical color filters for the imaging phase of the study were chosen at wavelength ranges that demonstrated reflectance ratios predominantly greater than or less than 1, and where filters were commercially available. The selected wavelength ranges are indicated in Fig. 2a with dashed vertical lines: green $(515-565 \mathrm{~nm})$, yellow (570 - 613 $\mathrm{nm})$, and red (604 - $644 \mathrm{~nm})$.

Representative images from stage two of the study can be seen in Fig. 3. Images of the surgical field taken with the (Fig. 3a) green, (Fig. 3b) yellow, and (Fig. 3c) red filters were used for quantitative evaluation of each filter. Figure $3 d$ shows the 

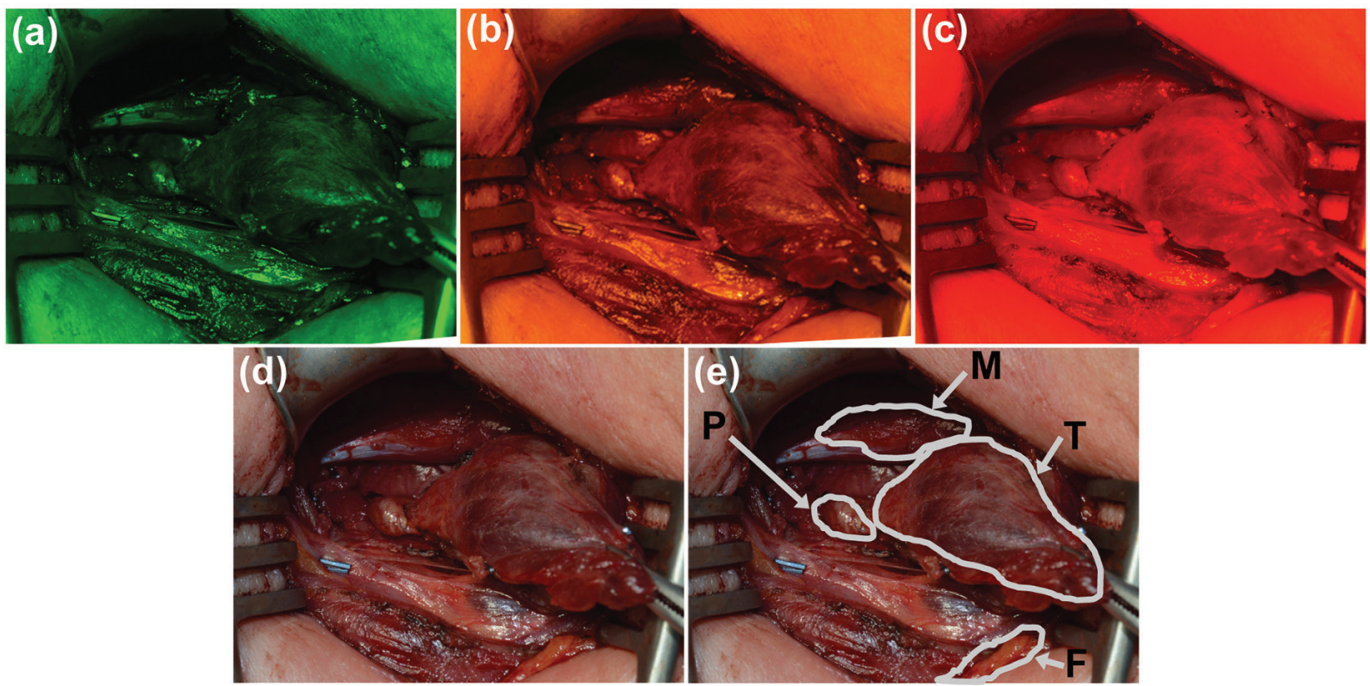

Figure 3. Representative images of a surgical field were taken with (a) green, (b) yellow, and (c) red narrow-band filters. (d) Image of the same field of view under white light and (e) with labels provided by the participating surgeon. P: parathyroid; M: muscle; T: thyroid; F: fat.

corresponding white light image of the surgical field, with each tissue type (parathyroid, thyroid, muscle, and fat) outlined by the surgeon in Fig. 3e.

The results of quantitative filter analysis can be seen in Fig. 4. The mean grayscale pixel value of each tissue type within the surgeon's outlined region was compared to that of the para- thyroid gland in the same subject. The mean reflectance ratios for all subjects are displayed as individual bars. Tissues that generated pixels brighter than the parathyroid gland have a ratio greater than 1 (black dashed line), while tissues that imaged darker than the parathyroid gland have a ratio less than 1 . None of these filters display a clear distinction between the parathy-

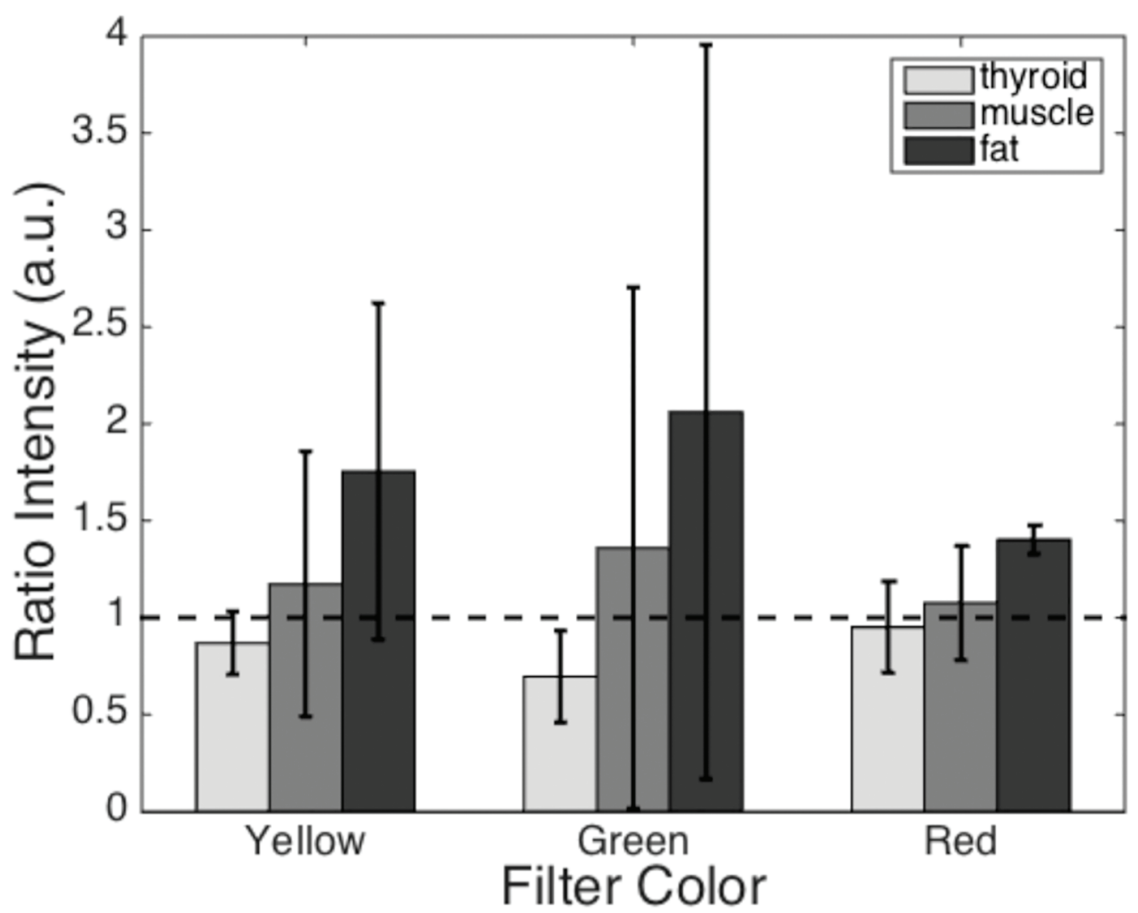

Figure 4. Mean reflected light intensity of each tissue type relative to the parathyroid gland intensity. The dashed line represents a ratio of one, where the intensity is equal to that of the parathyroid gland. Tissue/filter combinations with ratios greater than 1 will appear brighter than the parathyroid gland and tissues with ratios less than 1 will appear darker than the parathyroid gland. Error bars represent standard deviations for each dataset. 


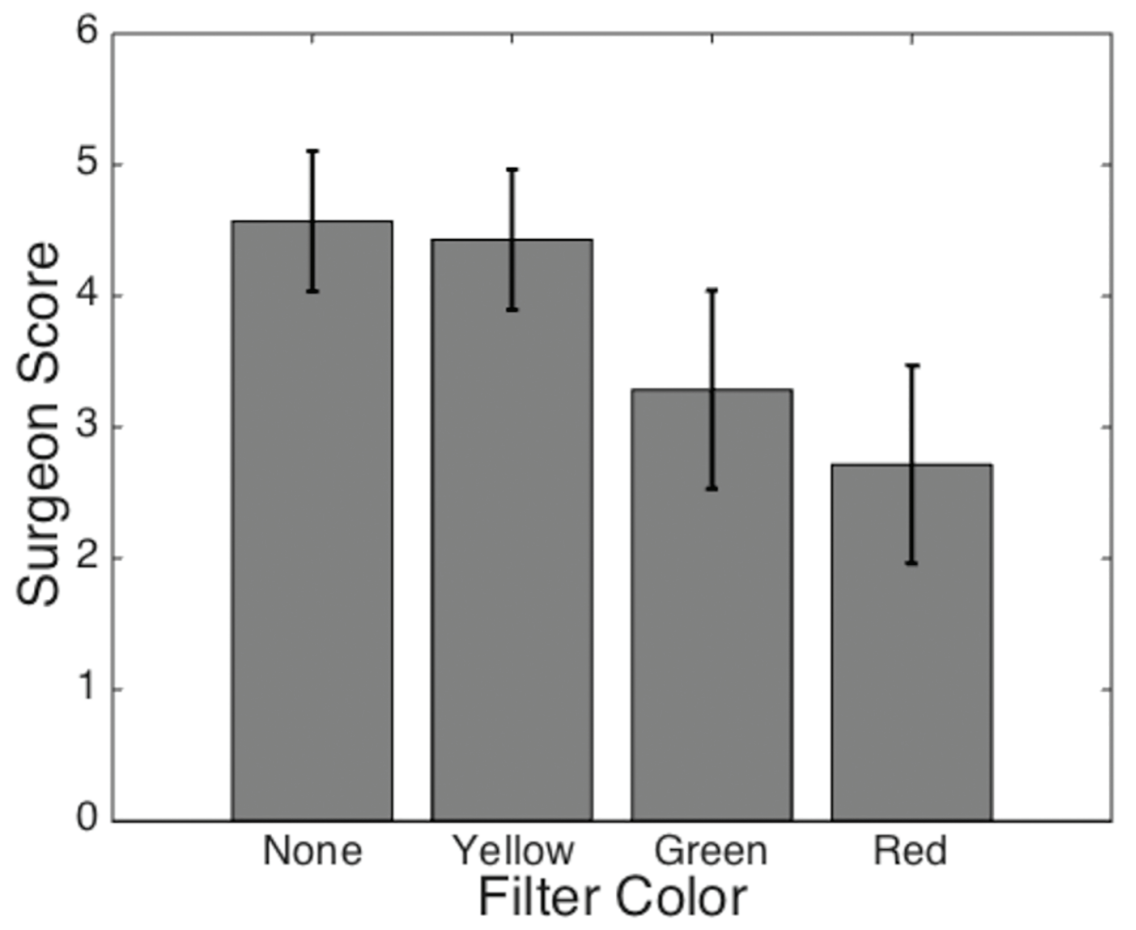

Figure 5. Average filter score based on surgeon's ability to distinguish the parathyroid gland from the surrounding tissue. The surgeon's confidence in identifying the parathyroid gland was scored on a scale from 1 (least confident) to 5 (most confident). Error bars represent standard deviations for each dataset.

roid gland and all of the other tissue types (ratio values much larger or smaller than 1).

The results of the qualitative assessment by the endocrine surgeon are seen in Fig. 5. The yellow filter was perceived to provide some improved subjective visualization as compared to the green or red filter. However, none of the filters demonstrated a definitive improvement relative to conventional white light examination.

\section{Discussion}

Intra-operative identification of the parathyroid gland can be challenging at times, even for the experienced endocrine surgeon. Pre-operative localization studies provide endocrine surgeons with an anatomic roadmap that helps allow for an efficient and safe minimally invasive parathyroidectomy [22]. These localization studies include the widely used ${ }^{99 \mathrm{~m}} \mathrm{Tc}$-sestamibi parathyroid nuclear scan, as well as neck ultrasound, and less commonly selective venous sampling, or rarely $4 \mathrm{D} \mathrm{CT}$ or MRI [3]. Localization studies are not foolproof and may fail to localize a parathyroid adenoma, may incorrectly identify a parathyroid adenoma, or may fail to identify a patient with multi-gland disease. Patients that fail to achieve a chemical cure after removal of a single parathyroid gland require further operative exploration with careful identification of each parathyroid gland.

Identification of parathyroid glands during thyroid surgery is equally as important so as to avoid injury to the parathy- roid glands with resultant hypoparathyroidism [9]. Even for the experienced endocrine surgeon, identification of small normal parathyroid gland during thyroid surgery can be difficult, especially in the setting of reoperative thyroid surgery or thyroiditis.

Certain intra-operative factors have been shown to accentuate and improve visualization of the parathyroid glands during surgery. Magnifying surgical telescopes (loupes) are widely used to assist in the identification of parathyroid glands [23]. Surgeon experience is an important factor in parathyroid surgery and has been positively correlated with successful identification of parathyroid glands [8]. Some have shown that methylene blue injection $[24,25]$ or radio-tracer injection [26] can improve intra-operative identification of the parathyroid glands during challenging cases. We hypothesized that given that the parathyroid glands have subtle but distinct tan-brown color (sometimes visualized as "mustard brown", "peanut butter brown", or "burgundy brown") and rounded, hyperemic, blanching, pulpy texture, they might be better visualized with simple and inexpensive optical filters placed over loupes that are already routinely used for these operations.

In order to test this hypothesis in a pilot study, wavelength bands (colors) with the largest differences in reflectance intensity between the parathyroid gland and other tissue types were first identified through diffuse reflectance spectroscopy measurements. Normalizing the reflectance spectra to each patient's own parathyroid gland accounted for natural patient-to-patient variation. The local maxima and minima that occur in the reflectance ratio data (Fig. 2a) correspond to the local maxima 
of oxy-hemoglobin absorption at 542 and $576 \mathrm{~nm}$ with a local minimum at $560 \mathrm{~nm}$ (Fig. 2b). These features may be a result of the differences in vasculature between the parathyroid gland and other tissues.

Optical filters selected in regions with large reflectance differences were evaluated for their ability to visibly enhance the identification of the parathyroid gland. We tested commercially available yellow, red, and green filters, each with a 40 - $50 \mathrm{~nm}$ bandwidth, based on these spectroscopy data and evaluated the results in a quantitative and qualitative manner. During image analysis, each tissue value was normalized to that of the corresponding parathyroid gland to minimize patient-to-patient variation. Although the reflectance ratios show some correlation with physiological properties of the tissue, the native variation among patients prevented use of one single filter color as a means of identifying the parathyroid gland. The standard deviations of these data show large variation across subjects. Averaging these ratios for each filter color and tissue type showed that none of the three filters could conclusively distinguish the parathyroid gland from each of the surrounding tissues.

The surgeon's qualitative evaluation of the filters was also considered in determining the utility of the selected optical filters. While the yellow filter was consistently ranked as the best of the three, it provided no perceived benefit over white light. The poor performance of the green filter may be a result of hemoglobin's high absorption at these wavelengths, causing tissues with high blood content to appear dark. This effect limited the surgeon's ability to use the subtle variations in tissue color and texture to identify the parathyroid gland. The overall appearance became dark and muted, illustrating the inherent tradeoff between specificity and brightness when selecting filter bandwidth. Larger bandwidth filters transmit more light providing a brighter visualization, but may not be able to highlight the differences that occur at discrete wavelengths. Because the goal of the study was to explore a method for parathyroid identification with limited interruption to the existing procedure, filters were selected with bandwidths slightly larger than those used for traditional NBI in an effort to minimize the additional hardware required for the procedure. These filters allowed for visualization using just the overhead surgical lights with the potential for future hardware development providing the surgeon with the ability to switch between white light and narrowband visualization.

One limitation of the study was that the measurements of the parathyroid glands were based on the surgeon's intra-operative identification of the gland in each patient, rather than the gold standard of surgical pathology. Although only those glands identified with high confidence under white light were included in data analysis, this means that the study can only evaluate how the technology compares to an imperfect standard.

Unfortunately, these filters failed to help enhance visualization of the parathyroid glands. In highlighting a specific color of light, narrow-band filters tend to reduce the overall light intensity, making it difficult to see. This study emphasizes that, in addition to the surgeon's experience and deep knowledge of anatomic variability of the parathyroid glands, it is not only color, but also texture, shape, variegation, and parathyroid location that contribute to correct identification of the parathyroid gland. These other visual cues were not improved by the optical filters.

\section{Conclusions}

In summary, NBI has previously proved valuable in enhancing the visual appearance of selected tissues in several clinical settings. Here, we applied a systematic two-stage approach to optimizing and evaluating NBI for surgical identification of the parathyroid gland. Simple color filters appear to offer little enhancement to assist parathyroid gland identification. Future studies may be considered exploring wide-band filters in the visible light range or other wavelengths in the infrared spectral region, or wavelengths emitted by targeted optical contrast agents to assist in the operative identification of parathyroid glands.

\section{Conflicts of Interest/Financial Disclosures}

The authors report no conflict of interest or proprietary or commercial interest in any product mentioned or concept discussed in this article.

\section{Author Contributions}

All authors (LMH, MCP, and TD) were involved in 1) the conception and design of the study, acquisition, analysis, and interpretation of data, 2) drafting and revising the article, and 3) final approval of the submitted version.

\section{References}

1. Marcocci C, Cetani F. Clinical practice. Primary hyperparathyroidism. N Engl J Med. 2011;365(25):2389-2397.

2. Bilezikian JP, Khan AA, Potts JT, Jr. Guidelines for the management of asymptomatic primary hyperparathyroidism: summary statement from the third international workshop. J Clin Endocrinol Metab. 2009;94(2):335-339.

3. Cheung K, Wang TS, Farrokhyar F, Roman SA, Sosa JA. A meta-analysis of preoperative localization techniques for patients with primary hyperparathyroidism. Ann Surg Oncol. 2012;19(2):577-583.

4. Norman J, Chheda H. Minimally invasive parathyroidectomy facilitated by intraoperative nuclear mapping. Surgery. 1997;122(6):998-1003; discussion 1003-1004.

5. Smith RB, Evasovich M, Girod DA, Jorgensen JB, Lydiatt WM, Pagedar NA, Spanos WC. Ultrasound for localization in primary hyperparathyroidism. Otolaryngol Head Neck Surg. 2013;149(3):366-371.

6. Starker LF, Mahajan A, Bjorklund P, Sze G, Udelsman R, Carling T. 4D parathyroid CT as the initial localization study for patients with de novo primary hyperparathyroidism. Ann Surg Oncol. 2011;18(6):1723-1728.

7. Lebastchi AH, Aruny JE, Donovan PI, Quinn CE, Callen- 
der GG, Carling T, Udelsman R. Real-Time Super Selective Venous Sampling in Remedial Parathyroid Surgery. J Am Coll Surg. 2015;220(6):994-1000.

8. Sosa JA, Bowman HM, Tielsch JM, Powe NR, Gordon TA, Udelsman R. The importance of surgeon experience for clinical and economic outcomes from thyroidectomy. Ann Surg. 1998;228(3):320-330.

9. Lin DT, Patel SG, Shaha AR, Singh B, Shah JP. Incidence of inadvertent parathyroid removal during thyroidectomy. Laryngoscope. 2002;112(4):608-611.

10. Lappas D, Noussios G, Anagnostis P, Adamidou F, Chatzigeorgiou A, Skandalakis P. Location, number and morphology of parathyroid glands: results from a large anatomical series. Anat Sci Int. 2012;87(3):160-164.

11. Suh H, A'Amar O, Rodriguez-Diaz E, Lee S, Bigio I, Rosen JE. Elastic light-scattering spectroscopy for discrimination of benign from malignant disease in thyroid nodules. Ann Surg Oncol. 2011;18(5):1300-1305.

12. Rosen JE, Goukassian ID, A'Amar OM, Bigio IJ and Lee SL. Early results of an in vivo trial of ess in thyroid cancer. SPIE BiOS International Society for Optics and Photonics. 2012;82071M-82071M-82011.

13. Briggs JC, A'amar O, Bigio I, Rosen JE, Lee SL, Sharon A, Sauer-Budge AF. Integrated device for in vivo fine needle aspiration biopsy and elastic scattering spectroscopy in preoperative thyroid nodules. J Med Devices. 2014;8(2):021003.

14. Conti de Freitas LC, Phelan E, Liu L, Gardecki J, Namati E, Warger WC, Tearney GJ, et al. Optical coherence tomography imaging during thyroid and parathyroid surgery: a novel system of tissue identification and differentiation to obviate tissue resection and frozen section. Head Neck. 2014;36(9):1329-1334.

15. Pitman MJ, Rosenthal JM, Savage HE, Yu G, McCormick SA, Katz A, Alfano RR, et al. The fluorescence of thyroid tissue. Otolaryngol Head Neck Surg. 2004;131(5):623627.

16. Paras C, Keller M, White L, Phay J, Mahadevan-Jansen A. Near-infrared autofluorescence for the detection of parathyroid glands. J Biomed Opt. 2011;16(6):067012.

17. McWade MA, Paras C, White LM, Phay JE, Mahadevan-
Jansen A, Broome JT. A novel optical approach to intraoperative detection of parathyroid glands. Surgery. 2013;154(6):1371-1377; discussion 1377.

18. Antakia R, Gayet P, Guillermet S, Stephenson TJ, Brown NJ, Harrison BJ, Balasubramanian SP. Near infrared fluorescence imaging of rabbit thyroid and parathyroid glands. J Surg Res. 2014;192(2):480-486.

19. Sharma P, Hawes RH, Bansal A, Gupta N, Curvers W, Rastogi A, Singh M, et al. Standard endoscopy with random biopsies versus narrow band imaging targeted biopsies in Barrett's oesophagus: a prospective, international, randomised controlled trial. Gut. 2013;62(1):15-21.

20. Garofolo S, Piazza C, Del Bon F, Mangili S, Guastini L, Mora F, Nicolai P, et al. Intraoperative narrow band imaging better delineates superficial resection margins during transoral laser microsurgery for early glottic cancer. Ann Otol Rhinol Laryngol. 2015;124(4):294-298.

21. Johnson KS, Chicken DW, Pickard DC, Lee AC, Briggs G, Falzon M, Bigio IJ, et al. Elastic scattering spectroscopy for intraoperative determination of sentinel lymph node status in the breast. J Biomed Opt. 2004;9(6):11221128.

22. Udelsman R, Lin Z, Donovan P. The superiority of minimally invasive parathyroidectomy based on 1650 consecutive patients with primary hyperparathyroidism. Ann Surg. 2011;253(3):585-591.

23. Testini M, Nacchiero M, Piccinni G, Portincasa P, Di Venere B, Lissidini G, Bonomo GM. Total thyroidectomy is improved by loupe magnification. Microsurgery. 2004;24(1):39-42.

24. Pollack G, Pollack A, Delfiner J, Fernandez J. Parathyroid surgery and methylene blue: a review with guidelines for safe intraoperative use. Laryngoscope. 2009;119(10):1941-1946.

25. Patel HP, Chadwick DR, Harrison BJ, Balasubramanian SP. Systematic review of intravenous methylene blue in parathyroid surgery. Br J Surg. 2012;99(10):1345-1351.

26. Somnay YR, Weinlander E, Alfhefdi A, Schneider D, Sippel RS, Chen H. Radioguided parathyroidectomy for tertiary hyperparathyroidism. J Surg Res. 2015;195(2):406411. 